doi:10.4149/neo_2013_022

\title{
Is there still a role for autologous stem cell transplantation in acute myeloid leukemia?
}

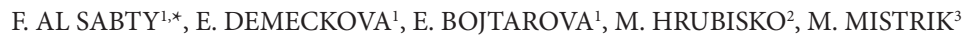 \\ ${ }^{1}$ Department of Hematology and Transfusion Medicine, University Hospital, Bratislava, Slovakia; ${ }^{2}$ Slovak Medical University, Bratislava, Slovakia; \\ ${ }^{3}$ Comenius University, Bratislava, Slovakia.
}

${ }^{*}$ Correspondence: alsabty@pe.unb.sk

Received August 8, 2012/ Accepted September 20, 2012

\begin{abstract}
Background: the role of autologous stem cell transplantation (ASCT) in treatment of acute myeloid leukemia (AML) remains unsettled. Aims: retrospective analysis to evaluate the role of ASCT in patients with AML without HLA-matched donor. Methods: between December 19, 1994 and August 1, 2012, a total of 63 patients with AML without HLA-matched donor in the department of Hematology and Transfusion Medicine, University Hospital, Bratislava, received an ASCT. Median age was 41 years (20-61 years). There were 35 (56\%) males and 28 (44\%) females. At the time of ASCT, 50 (79\%) patients were in first complete remission (CR), 11 (18\%) patients were in second CR and 2 (3\%) patients were in relapse. Results: with a median follow-up of 115 months (34-214 months), the 10 year overall survival (OS) and disease free survival (DFS) of all patients was $55 \%$ and $51 \%$, respectively. Transplant-related mortality was $6 \%$. The relapse rate was $38 \%$ and 9 years probability of relapse was $44 \%$. Conclusion: ASCT is still an effective post-remission treatment in AML patients without HLA-matched donor; with the possibility of long-term survival or even cure in remarkable proportion of patients with AML, particularly in patients with favorable and intermediate cytogenetic risk.
\end{abstract}

Key words: acute myeloid leukemia, autologous stem cell transplantation, survival, treatment

In the last two decades, the treatment of acute myeloid leukemia (AML) has evolved with the use of more aggressive therapeutic strategies [1]. Post-remission treatment remains a critical issue in this disease $[1,2]$. During the last years, autologous transplantation has emerged as another promising approach to improve post-remission therapy of AML [3]. Autologous transplant offers a way to use the antileukemia effectiveness of ablative therapy without the morbidity and mortality of graft-versus-host disease that complicates allogeneic transplant. In addition to improved safety, autologous transplant is also more broadly applicable, potentially allowing the treatment of all patients who achieve remission and extending the age of transplant to 60 or even 70 years. Despite a wealth of phase II studies as well as some large phase III studies, the role of autologous transplant in the treatment of patients with AML in first remission remains unsettled. A number of phase II studies have produced outcomes that appear superior to those achievable with standard chemotherapy, including high dose Ara-C [3]. However, the results of phase III studies have been interpreted by some to minimize the role of upfront autologous transplant in first remission. These phase III studies were designed and implemented before the importance of prognostic features, especially cytogenetics, were fully appreciated. Based on our current understanding of heterogeneity of AML and the need to approach treatment with a risk-adapted method, the question no longer appears to be 'What is the best treatment for AML?' but rather determining the best treatment approach for each category of AML. In this retrospective study, we report the results of patients with good, standard and high-risk AML who underwent ASCT in our institution over the last 17 years. We analyzed the effect of several important prognostic factors on overall survival (OS) and disease free survival (DFS), relapse and transplant-related mortality. Furthermore we compared the outcome with the expected outcome based on previous international studies.

\section{Materials and methods}

Patients: ASCT has been used in our institution as post-remission therapy for all patients less than 60 years old (between 
Table 1. Patient characteristics

\begin{tabular}{|c|c|}
\hline Number of patients & 63 \\
\hline Median age (range) & $41(20-61)$ \\
\hline \multicolumn{2}{|l|}{ Sex } \\
\hline Male & $35(56 \%)$ \\
\hline Female & $28(44 \%)$ \\
\hline \multicolumn{2}{|l|}{ AML subtypes } \\
\hline M0 & $1(2 \%)$ \\
\hline M1 & $4(6 \%)$ \\
\hline M2 & $36(57 \%)$ \\
\hline M4 & $16(25 \%)$ \\
\hline M5 & $6(10 \%)$ \\
\hline Secondary AML & $12(19 \%)$ \\
\hline \multicolumn{2}{|l|}{ Cytogenetic risk group } \\
\hline Good & $5(8 \%)$ \\
\hline Intermediate & $43(68 \%)$ \\
\hline Poor & $10(16 \%)$ \\
\hline Unknown & $5(8 \%)$ \\
\hline \multicolumn{2}{|l|}{ ECOG-WHO performance status } \\
\hline 0 & $28(44 \%)$ \\
\hline 1 & $22(35 \%)$ \\
\hline 2 & $5(8 \%)$ \\
\hline 3 & $2(3 \%)$ \\
\hline Unknown & $6(10 \%)$ \\
\hline \multicolumn{2}{|l|}{ WBC at diagnosis } \\
\hline Median x $10^{9} / 1$ (range) & $17(1-170)$ \\
\hline Patients with WBC $\leq 10 \times 10^{9} / 1$ & $23(37 \%)$ \\
\hline Patients with WBC $>10<100 \times 10^{9} / 1$ & $28(44 \%)$ \\
\hline Patients with WBC $\geq 100 \times 10^{9} / 1$ & $4(6 \%)$ \\
\hline Unknown & $8(13 \%)$ \\
\hline \multicolumn{2}{|l|}{ Disease state at ASCT } \\
\hline CR1 & $50(79 \%)$ \\
\hline CR2 & $11(18 \%)$ \\
\hline Relapse & $2(3 \%)$ \\
\hline \multicolumn{2}{|l|}{ AML risk } \\
\hline Standard risk & $37(59 \%)$ \\
\hline High risk & $26(41 \%)$ \\
\hline Days from CR to ASCT (range) & $107(48-281)$ \\
\hline$<3$ months & $21(33 \%)$ \\
\hline$>3$ months & $30(48 \%)$ \\
\hline Unknown & $12(19 \%)$ \\
\hline \multicolumn{2}{|l|}{ Number of cycles prior to ASCT } \\
\hline 1 & $29(46 \%)$ \\
\hline 2 & $15(24 \%)$ \\
\hline 3 & $2(3 \%)$ \\
\hline Unknown & $17(27 \%)$ \\
\hline \multicolumn{2}{|l|}{ Source of graft } \\
\hline PBSC & $53(84 \%)$ \\
\hline BMSC & $5(8 \%)$ \\
\hline $\mathrm{PBSC}+\mathrm{BMSC}$ & $5(8 \%)$ \\
\hline \multicolumn{2}{|l|}{ Conditioning regimens } \\
\hline BUMEL & $53(84 \%)$ \\
\hline BUCY2 & $3(5 \%)$ \\
\hline $\mathrm{BU}$ & $3(5 \%)$ \\
\hline E-BUCY2 & $3(5 \%)$ \\
\hline E-BU & $1(1 \%)$ \\
\hline
\end{tabular}

18-60 years) with AML (except M3) in first CR who lacked an HLA-matched donor. Patients with more advanced disease $(\geq$ second CR) and without an HLA-matched donor, regardless of their cytogenetic profile, were also submitted to an ASCT. All patients were stratified into three risk groups; poor, intermediate and good-risk groups on the basis of cytogenetic and molecular analyses at diagnosis [2]. Between December 19, 1994 and August 1, 2012, a total of 63 patients with AML in the Department of Hematology and Transfusiology, University Hospital, Bratislava, received ASCT. All patients gave written informed consent. The clinical characteristics of the patients are depicted in Table 1.

Treatment plan: The induction treatment consisted of one course, or in the case of a partial response, two courses of chemotherapy (CHT). All patients who had a complete remission received 1-3 courses of intensive consolidation CHT. Patients with a confirmed complete remission and no HLA-identical donor were scheduled for ASCT.

Mobilization and stem cell collection: Following the first or the second consolidation CHT, granulocyte colony stimulating factor (G-CSF; Filgrastim or Lenograstim) was administered from day 11 after the end of consolidation CHT at a dose $5 \mu \mathrm{g} / \mathrm{kg} / \mathrm{day}$, and continued until PBSC harvest. The full blood count was monitored daily until regeneration (WBC $>4 \times 10^{9} / 1$ ), then CD34 $4^{+}$cells were measured via flow cytometry and leukapheresis was commenced when absolute $\mathrm{CD} 34^{+}$cell

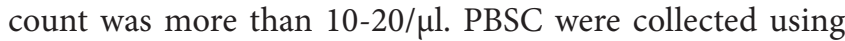
cell separator (mostly Cobe Spectra), 3-5 times total patient blood volume in a single or 2 (rarely 3 ) aphaeresis procedures. Eligibility to proceed to high-dose therapy and transplant was defined by collection of at least $1 \times 10^{6} / \mathrm{kg} \mathrm{CD} 34^{+}$cells. The PBSC product was cryopreserved with with $8-10 \%$ DMSO in liquid nitrogen at $-196^{\circ} \mathrm{C}$. No in vitro purging procedure was performed. Thawing was performed rapidly at $37^{\circ} \mathrm{C}$ on the day of the transplant and / the cells were immediately infused intravenously. CD $34^{+}$and colony-forming unit granulocyte-macrophage (CFU-GM) assays were done according to institutional criteria. Patients who failed to achieve adequate harvest quality/yield were offered a second course of mobilization CHT with cyclophosphamide $2-3 \mathrm{~g} / \mathrm{m}^{2}$ iv infusion in 2 hours as single dose then G-CSF was given after 7 days in the same dose as above until PBSC harvest. If PBSC harvest failed after the second course of CHT then mobilization was stopped. In poor mobilizers bone marrow harvesting was done under general anesthesia.

Source of graft. Transplantation of bone marrow progenitor cells (BMSC) was performed in 5 patients $(8 \%)$ whereas in 53 patients (84\%) peripheral blood stem cells (PBSC) were used. The remaining 5 patients (8\%) underwent transplantation with a combination of BM and PBSC due to a low CD34 $4^{+}$ cell count obtained in the aphaeresis.

Conditioning regimen and autologous stem cell transplantation. The patients were treated with two different protocols, 1) busulfan $(\mathrm{Bu}) 4 \mathrm{mg} / \mathrm{kg} /$ day, p.o. every 6 hours for 4 days (total dose $16 \mathrm{mg} / \mathrm{kg}$ ) on days -6 through 
-3 and melfalan (Mel) $140 \mathrm{mg} / \mathrm{m}^{2}$ iv for 1 day on days -2 (BuMel)( $\mathrm{n}=53 ; 84 \%) .2$ ) busulfan (Bu) $4 \mathrm{mg} / \mathrm{kg} /$ day p.o. every 6 hours for 4 days (total dose $16 \mathrm{mg} / \mathrm{kg}$ ) on days -7 through -4 followed by cyclophosphamide $60 \mathrm{mg} / \mathrm{kg} /$ day iv for 2 days (total dose $120 \mathrm{mg} / \mathrm{kg})$, on days -3 and $-2(\mathrm{BuCy} 2)(\mathrm{n}=3 ; 5 \%)$. In 3 patients (5\%), the protocol regimen was reduced due to the expected toxicity; they received only busulfan (Bu) $4 \mathrm{mg} /$ $\mathrm{kg} /$ day p.o. every 6 hours for 4 days (total dose $16 \mathrm{mg} / \mathrm{kg}$ ). In 3 patients (5\%), the protocol regimen was intensified by the addition of etoposide (VP-16) $60 \mathrm{mg} / \mathrm{kg}$ iv on day -3 (EBuCy2), and 1 patient (1\%) received E-Bu. PBSC or BMSC were infused on day 0 . A median number of total nucleated cells of $6.2 \times 10^{8} / \mathrm{kg}$ (range $1.35-38.9$ ), CD34 ${ }^{+}$cells of 2.6 x $10^{6} / \mathrm{kg}$ (range $0.52-32.5$ ) and CFU of $82 \times 10^{4} / \mathrm{kg}$ (range $2.4-1463$ ) had been reinfused. All patients received G-CSF $5 \mu \mathrm{g} / \mathrm{kg} /$ day (from day +11 ) and erythropoietin $10000 \mathrm{IU} /$ day (from day +12 ) until hemopoietic recovery. Low molecular weight heparin was used as prophylaxis of hepatic venooclusive disease.

Supportive care. Patients were isolated in high-efficiency particulate air-filtered positive-pressure air-flow rooms during the period of pancytopenia associated with the conditioning regimen and after ASCT. All patients received prophylactic red cell and platelet transfusions to maintain hemoglobin concentration more than $90 \mathrm{~g} / \mathrm{l}$ and platelet count more than $10-20 \times 10^{9} / 1$. Antimicrobial prophylaxis was not routinely given. Febrile neutropenia was treated according to the local guidelines for treatment of febrile neutropenia.

Statistical analysis. Data were analyzed as of August 1, 2012. All patient details were routinely introduced and analyzed with the SPSS statistical software versions 16.0, 2008. Primary end points were engraftment, treatment-related mortality (TRM), relapse, DFS and OS. Survival curves were plotted following the Kaplan-Meier method and differences between the curves were analyzed with the log-rank test. Potential prognostic factors were tested by regression analysis using linear regression for engraftment and Cox proportional hazard model for DFS, OS, TRM, and relapse. Two-tailed $\mathrm{P}$ values with significance of $<0.05$ have been used.

\section{Results}

Engraftment. Overall, 60 of 63 patients (95\%) achieved a sustained granulocyte count greater than $/ 0.5 \times 10^{9} / 1$ with a median of $13(10-37)$ days and a sustained leukocyte count greater $1 \times 10^{9} / 1$ with a median of $13(9-37)$ days after transplantation. In 54 of $63(86 \%)$ patients, the median time to a self-sustained platelet count higher than 20 and $50 \times 10^{9} / 1$ was $30(10-130)$ and $53(10-190)$ days, respectively. The patients not included in these results either died or had an early relapse before achieving the mentioned neutrophil and platelet counts. The time to engraftment depended on the source of stem cells and it was faster in recipients of PBSC. The patients transplanted with BM, PBSC and the combination of BM plus PBSC attained a neutrophil count greater than $0.5 \times 10^{9} / 1$ with a median of 24 ,
13 and 13 days post-transplant, respectively $(P=0.034)$. Similarly, the same subgroups of patients achieved a platelet count greater than $20 \times 10^{9} / 1$ with a median of 66,29 and 20 days after the stem cell infusion, respectively $(P=0.076)$.

Transplant-related mortality. Overall, 4 of 63 (6\%) patients died with transplant-related causes; all 4 patients were in CR1. Two patients in the BuMel arm died within 100 days of transplant: 1 with GIT toxicity at day +35 and 1 with hemorrhage at day +38 . One patient treated with E-Bu died day +9 because of sepsis and MODS. One patient treated with Bu died day +15 also because of sepsis and MODS. Using the regression analysis tests we did not found any significant prognostic factor that may affect TRM.

Relapse rate and the cumulative incidence of relapse. Of the 61 patients who underwent ASCT in CR1 or CR2, 23 patients (38\%) relapsed. Median time from CR to relapse was 46 weeks (range 27-284 weeks). Median time to relapse after ASCT was 32 weeks (range 12-268 weeks). The majority of patients (81\%) relapsed at first 60 weeks ( 8 months). 4 patients (7\%) were missing. 2 patients who were transplanted at early relapse did not achieve CR and they died because of leukemia progression. The cumulative incidence of relapse (CIR) at 9 years ( 9 years probability of relapse) was $44 \% \pm 2.7$, for the entire group of patients. (Figure $1 \mathrm{C}$ ).

Overall survival (OS) and disease free survival (DFS). At the time of analysis, the survival state of $58 / 63$ patients (92\%) were known, 5 patients (8\%) were lost to follow up and they are censored at the time of the last contact. Overall, 24 patients (38\%) died before the date of analysis. 4 patients (6\%) died at $\mathrm{CR}$, whereas in the remaining patients leukemia progression was the direct cause of death. The remaining 34 (54\%) patients who are still alive had a median follow-up time of 92 months (range 11 - 191 months).

The 10 years Kaplan-Meier estimated probabilities of overall survival (OS) and disease free survival (DFS) after ASCT for the whole group of patients was $55 \%$ and $51 \%$, respectively. (Figure $1 \mathrm{~A}, \mathrm{~B}$.). When adjusted according to cytogenetic risk groups the 10 year probabilities of OS and DFS in patients with good and intermediate cytogenetic risk groups was $60 \%$ and $56 \%$ respectively, compared to $31 \%$ and $27 \%$ in patients with poor cytogenetic risks. (Figure 2).

Prognostic factors effect on outcome (OS and DFS) and relapse. The impacts of clinical variables that have been reported to be potential prognostic factors predictive of outcome had been analyzed by univariate and multivariate analysis. Three factors emerged as statistically significant for survival in univariate analysis: WBC count at diagnosis, ECOG performance status and cytogenetic risk. Whereas, for relapse only two factors emerged as statistically significant: WBC count at diagnosis and cytogenetic risk. (Table 2).

There was a strong impact of the cytogenetic risk group on the probability of OS, DFS and relapse. Patients with a favorable and intermediate cytogenetic risk had a significantly superior OS ( $60 \%$ vs. $31 \%, \mathrm{P}=0.05)$, DFS ( $56 \%$ vs. $27 \%, \mathrm{P}=0.05$ ) and lower incidence of relapse (CIR $40 \%$ vs. 

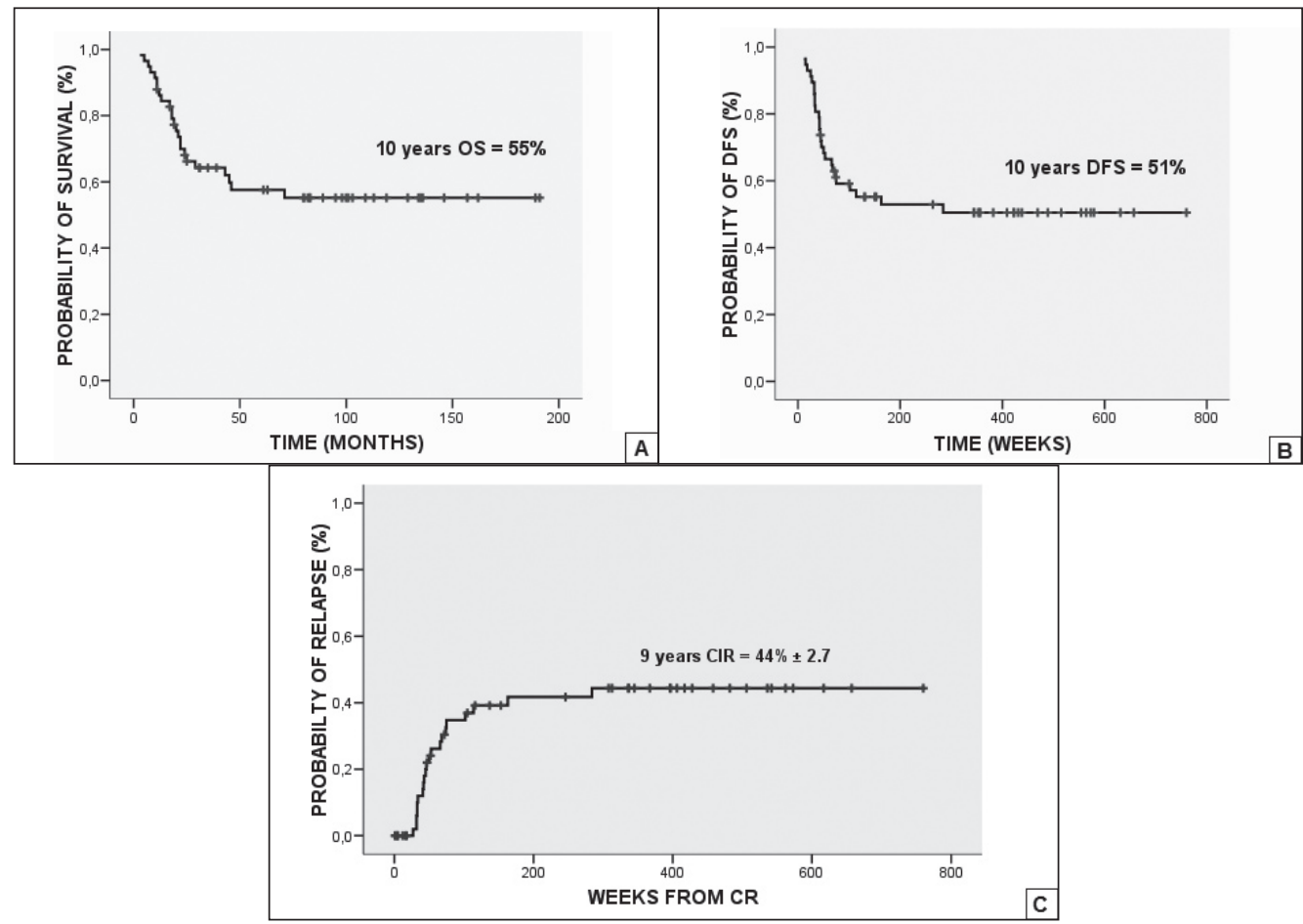

Figure 1. A, B: The 10 years Kaplan-Meier estimated probabilities of overall survival (OS) and disease free survival (DFS) after ASCT for the whole group of patients C: Cumulative incidence of relapse (CIR) at 9 years for the whole group of patients.

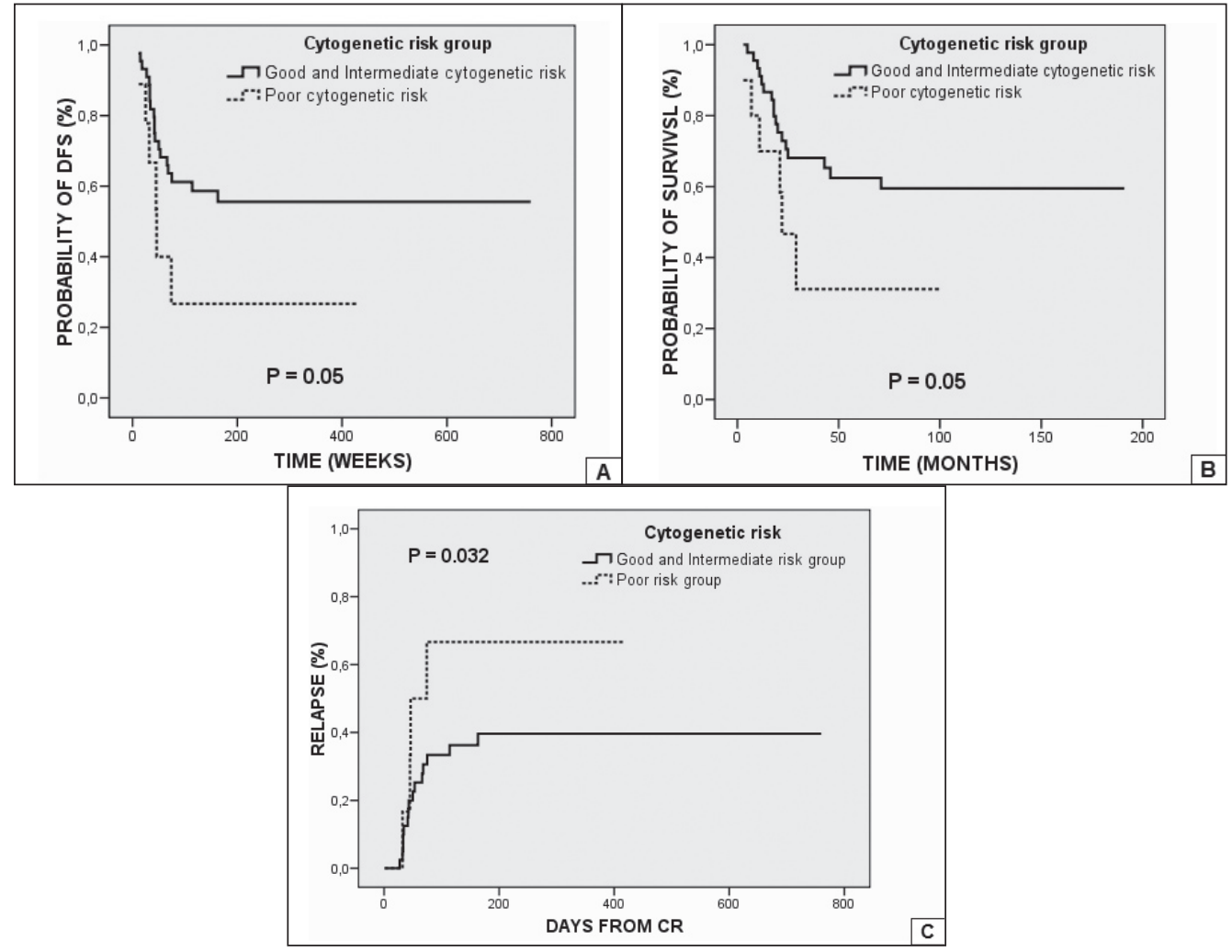

Figure 2. The impact of cytogenetics on the outcome; probability of A ) DFS, B) OS and C) relapse in good and intermediate vs. poor cytogenetic risk groups 
Table 2. Univariate and multivariate analysis of prognostic factors of OS, DFS and relapse.

\begin{tabular}{|c|c|c|c|c|c|c|}
\hline \multirow[b]{2}{*}{ Risk factors } & \multicolumn{2}{|c|}{ DFS } & \multicolumn{2}{|c|}{ OS } & \multicolumn{2}{|c|}{ Relapse } \\
\hline & $\begin{array}{c}\text { Univariate } \\
P \text { Value }\end{array}$ & $\begin{array}{c}\text { Multivariate } \\
P \text { Value }\end{array}$ & $\begin{array}{c}\text { Univariate } \\
P \text { Value }\end{array}$ & $\begin{array}{c}\text { Multivariae } \\
P \text { Value }\end{array}$ & $\begin{array}{c}\text { Univariate } \\
P \text { Value }\end{array}$ & $\begin{array}{c}\text { Multivariae } \\
P \text { Value }\end{array}$ \\
\hline Age & 0.62 & 0.10 & 0.35 & 0.2 & 0.44 & 0.49 \\
\hline Sex & 0.16 & 0.09 & 0.46 & 0.09 & 0.82 & 0.79 \\
\hline AML subtype & 0.31 & 0.29 & 0.17 & 0.49 & 0.67 & 0.41 \\
\hline Cytogenetic risk & 0.05 & 0.35 & 0.05 & 0.62 & 0.032 & 0.27 \\
\hline WBC at diagnosis & 0.05 & 0.77 & 0.12 & 0.79 & 0.056 & 0.69 \\
\hline WHO performance & 0.042 & 0.094 & 0.09 & 0.42 & 0.062 & 0.30 \\
\hline AML risk group & 0.10 & 0.58 & 0.11 & 0.47 & 0.08 & 0.57 \\
\hline Source of graft & 0.75 & 0.87 & 0.78 & 0.81 & 0.31 & 0.92 \\
\hline No. of CD34 ${ }^{+}$cells infused & 0.29 & 0.24 & 0.39 & 0.67 & 0.37 & 0.99 \\
\hline No. of nucleated cells & 0.54 & 0.43 & 0.18 & 0.45 & 0.71 & 0.59 \\
\hline No. of CFU infused & 0.43 & 0.56 & 0.38 & 0.53 & 0.52 & 0.95 \\
\hline Conditioning regimen & 0.09 & 0.91 & 0.22 & 0.49 & 0.69 & 0.92 \\
\hline Disease state at ASCT & 0.57 & 0.87 & 0.19 & 0.98 & 0.67 & 1 \\
\hline Time from CR to ASCT & 0.70 & 0.08 & 0.81 & 0.31 & 0.64 & 0.72 \\
\hline No. of cycles prior to ASCT & 0.30 & 0.74 & 0.11 & 0.37 & 1 & 0.48 \\
\hline HD-AraC in induction & 0.77 & 0.98 & 0.75 & 0.47 & 0.65 & 0.078 \\
\hline HD-AraC in consolidation & 0.35 & 0.76 & 0.76 & 0.88 & 0.54 & 1 \\
\hline
\end{tabular}

$67 \%, \mathrm{P}=0.032)$ than those with unfavorable cytogenetic risk (Figure 2). Patients with WBC count $<10 \times 10^{9} / 1$ at diagnosis had significantly better DFS $(68 \%$ vs. $35 \%, \mathrm{P}=0.05)$ and lower incidence of relapse (CIR $26 \%$ vs. $60 \%, \mathrm{P}=0.056$ ) than patients with WBC count $>10 \times 10^{9} / 1$. Patients with grade 0 ECOG performance state tolerate therapy well with significant improvement in outcome compared with patients with more advanced grades of ECOG performance state, (OS $72 \%$ vs. $40-50 \%, \mathrm{P}=0.09$ and DFS $66 \%$ vs. $20-50 \%, \mathrm{P}=0.04)$. There was a non-significant trend toward improved outcome for patients aged $<40$ compared with those $>40$ years, with OS $60 \%$ vs. $52 \%$, respectively. Females also had non-significantly better outcome and less relapse than males, with OS 61\% vs. $50 \%$; DFS $63 \%$ vs. $39 \%$ and CIR $30 \%$ vs. $58 \%$, respectively. We also observed a trend for a better DFS in patients treated with the BuCy2 regimen in comparison with those conditioned with BuMel $(67 \%$ vs. $51 \%, \mathrm{P}=0.09)$. There was also a nonsignificant trend toward improved outcome for those who received two cycles of consolidation chemotherapy prior to autologous SCT compared with those who received only one cycle (OS 63\% vs. 58\%; DFS 59\% vs. 55\%). All patients $(n=2)$ who were transplanted at relapse died 2 months and 18 months after autologous SCT. Patients who were transplanted in CR1 $(n=50)$ had a non-significantly better OS and DFS compared with those patients who were transplanted in CR2 ( $\mathrm{n}=11)$ (OS $60 \%$ vs. $50 \%, \mathrm{P}=0.19$; and DFS $54 \%$ vs. $43 \%, \mathrm{P}=0.57$ ).

\section{Discussion}

Intensive combination $\mathrm{CHT}$ as primary treatment of AML leads to a CR in the majority of the patients (60-80\%) [2]. However, despite subsequent treatments intended to maintain such remission, most patients relapse within 2 to 3 years of initial presentation [1]. In the last two decades, ASCT has been used to consolidate a substantial number of patients with AML who do not have an HLA-matched related or unrelated donor [1,

Table 3. Comparison of our results with three large phase III randomized studies, ALWP-EBMT registry and two recent long-term single institution studies.

\begin{tabular}{|c|c|c|c|c|c|c|}
\hline Refs. & Pat. No. & Years & OS $\%$ & DFS \% & RR \% & TRM\% \\
\hline Zittoun $^{7}$ (EORTC/GIMEMA) & 95 & 4 & 56 & 48 & 41 & 9 \\
\hline Burnett $^{8}$ (MRC-10) & 126 & 7 & 57 & 54 & 35 & 12 \\
\hline Cassileth $^{9}$ (ECOG/ Intergroup) & 63 & 4 & 43 & 35 & 48 & 14 \\
\hline ALWP-EBMT registry ${ }^{10}$ & 2100 & 5 & 51 & 43 & 53 & 9 \\
\hline Roberto M. ${ }^{4}$ & 118 & 10 & 54 & 50 & 46 & 4 \\
\hline Carols M. ${ }^{1}$ & 42 & 13 & 52 & 40 & 48 & 14 \\
\hline Our study & 63 & 10 & 55 & 51 & 38 & 6 \\
\hline
\end{tabular}


3-6]. The role of ASCT in AML remains controversial. Phase II and III trials showed that ASCT provides lower relapse rates than conventional CHT and improves DFS in some but not in all studies $[3,7-9]$. Our retrospective study analyzed the role of ASCT in patients with AML, with good, intermediate and poor prognostic features. Although this study has a small number of patients, it shows long-term results with 9.5 years of median follow-up. Therefore, patients not relapsing at this late timing post-transplant have a high likelihood of being cured. In table 3 we compared our results with three large phase III randomized studies [7-9], ALWP-EBMT registry [10] and two recent long-term single institution studies $[1,4]$.

It is now well proven that hemopoiesis recovery (engraftment) is more rapid with PBSCT than with BMSCT [11-13]. The most important factor for hemopoietic recovery after ASCT is the dose of progenitor cells infused [11-13]. In our study the engraftment after PBSCT was significantly shorter than after BMSCT by 11 days (for neutrophils recovery) and by 37 days (for platelets recovery), and it was comparable with other series. This difference is due to the fact that the dose of progenitor cells infused in PBSCT was significantly higher than in BMSCT. There was no statistical difference in the dose of $\mathrm{CD} 34^{+}$cells infused in patients with PBSCT and BMSCT $(P=0.909)$, but the difference was in total nucleated cells and CFU ( $P=0.002$ a 0.001 respectively). We also observed a strong correlation between $\mathrm{CFU}$ and engraftment (Spearman's test; $P=0.002$ and 0.044 for neutrophil a platelets recovery, respectively), therefore CD34+ cells alone is a poor indicator for the target dose of progenitor cells to be infused. The TRM observed in our group was $6 \%$ which is lower than in other studies; probably due to better supportive therapy. In our study the relapse rate (38\%) and the cumulative incidence of relapse at 9 years (44\%) was lower than in other studies. The 10 years probabilities of OS (55\%) and DFS (51\%) after ASCT for the whole group of patients were comparable and even better than in other studies. Overall, the results obtained in patients with good and standard cytogenetic risk are quite encouraging and clearly superior to the results achieved with $\mathrm{CHT}$ alone post-remission in other institutions $[2,8,14-16]$. However, the results in patients with high cytogenetic risk disease were dismal and not superior to published results of $\mathrm{CHT}$ alone after attaining CR. [1, 2, 8, 14-16]. Therefore, patients with poor cytogenetic prognosis AML lacking an HLA-matched donor should probably be offered an allogeneic SCT from an alternative unrelated donor or from a haplotype-mismatched related donor. We found a different in survival pattern dependent on the initial leukocyte count. Patients with WBC count $<10 \times 10^{9} / 1$ at diagnosis had significantly better OS and DFS with less incidence of relapse compared to patients with WBC count $>10 \times 10^{9} / 1$. Patients with good ECOG performance state did well during ASCT with significant improvement in outcome. One of major biases of retrospective studies of patients who undergo transplantation is the time-censoring effect, that is, patients who are transplanted late after the achievement of CR may be at low risk for relapse [1]. In our study, $33 \%$ and $48 \%$ patients were autografted within $<3$ months and > 3 months from CR respectively; with no difference in outcome. Since our study extends over 17 years, some of our protocols changed according to the international tendencies. This is the reason why there is some heterogeneity regarding the induction $\mathrm{CHT}$, consolidation $\mathrm{CHT}$, source of stem cells and the use of G-CSF post-transplant. Despite the heterogeneity of therapeutic regimens, there was no difference in outcome based on induction or consolidation $\mathrm{CHT}$ received. Furthermore the incorporation of $\mathrm{HD}-\mathrm{AraC}$ in the induction or consolidation CHT did not have any effect on the outcome, or relapse. In our series, although the recipients of PBSC and of G-CSF post-transplant had rapid engraftment, and hence fewer days of fever, of intravenous antibiotics and of hospitalization days, we did not observe any impact on their survival or relapse patterns. Age is identified as a significant prognostic factor for survival and it is clear from a number of studies that the benefits of intensified therapy in AML are confined to younger patients [2]. In our study we also observed a better outcome in younger patients $<40$ years compared to those $>40$ years, but this was statistically non-significant. Similarly, we found a non-significantly higher rate of survival and lower incidence of relapse of females in relation to males, although the ratio of male to female was $1.25: 1$, this can be explained by the fact that in male group there was more patients with poor cytogenetic ( $60 \%$ vs. $40 \%)$ and high WBC count (63\% vs. $38 \%$ ) compared to females. Furthermore 2 male patients were transplanted in relapse (then subsequently died) whereas all females were transplanted in CR1 or CR2. The number of CHT cycles prior to ASCT ( 2 vs.3) were strong predictors of both survival and relapse $[4,10,17,18]$. In our study there was a non-significant trend towards improved outcome for those who received two cycles of consolidation CHT prior to ASCT compared with those who received only one cycle. Therefore, based on our results, two courses of consolidation in AML (effective in vivo purging) is advisable if the patient is going to be submitted to an ASCT. Although the BuCy2 regimen appeared to have been superior to the BuMel regimen in our study, it did not attain statistical significance. No formal, randomized assessment of the role of cyclophosphamide in the ASCT has been undertaken.

In summary, despite the lack of a graft-versus-leukemia effect, ASCT is a recognized therapeutic option for AML patients without HLA matched donor. Our data are consistent with others reports showing the ability of ASCT to generate long-term DFS or even cure in a significant proportion of patients with AML, particularly in those with good or standard risk disease.

\section{Conclusion and future directions}

ASCT is an important treatment modality in the treatment of AML and interest in this area remains high. Appropriate patient selection based on a risk adapted strategy is important for the proper application or study of this treatment modality. Patients with favorable cytogenetics appear to derive significant benefit whereas patients with very high-risk cytogenetics should preferentially be treated with allogeneic transplant when this modality is available. Outside the setting of clinical trials, ASCT 
for intermediate-risk patients is an appropriate treatment choice and seems to improve outcomes compared to conventional CHT. The choice between autologous and allogeneic transplant in this patient group involves consideration of several factors including age, performance status, donor availability, and risk-assessment of the leukemia based on factors other than cytogenetics. Autologous PBSCT is likely to replace autologous BMT based on reduction in morbidity, resource utilization, and duration of hospitalization. Post-remission consolidation therapy prior to stem cell collection appears to be very important. The optimal nature and duration of consolidation prior to stem cell collection remain to be determined. Even with the in vivo purging based on prior cytoreduction therapy, the risk of leukemia contamination of stem cells remains. Hopefully effective and nontoxic methods of in vitro purging will be developed.

Acknowledgement: Foremost, I would like to thank transplantation staff in our hospital for the great job in the management of hemato-oncological patients. Furthermore I would like to thank the data manager and all the peoples who help me in preparing this article.

\section{References}

[1] MARTINS C, LACERDA JF, LOURENÇO F, CARMO JA, LACERDA JMF. Autologous stem cell Transplantation in acute myeloid Leukemia: Factors influencing outcome. A 13 year single institution experience. Acta Med Port 2005; 18: 329-338.

[2] DOHNER H, ESTEY EH, AMADORI S, APPELBAUM FR, BUCHNER T, et al. Diagnosis and management of acute myeloid leukemia in adults: recommendations from an international expert panel, on behalf of the European LeukemiaNet. Blood. 2010; 115: 453-474. http://dx.doi.org/10.1182/blood2009-07-235358

[3] LINKER CA. Autologous stem cell transplantation for acute myeloid leukemia. Bone Marrow Transplantation 2003; 31, 731-738. http://dx.doi.org/10.1038/sj.bmt.1704020

[4] LEMOLI RM, BACCARANI M, ISIDORI A, BONIFAZI F, VISANI G, et al. Autologous stem cell transplantation for acute myeloid leukemia patients in first complete remission: a 10 -year follow-up study of 118 patients. Haematologica 2005; 90: 139-141.

[5] BREEMS DA, LOWENBERG B., Acute myeloid leukemia and the position of autologous stem cell transplantation. Semin Hematol 2007; 44: 259-266. http://dx.doi.org/10.1053/ j.seminhematol.2007.08.002

[6] EWING JC, ROBERTSON JD, KELL WJ, BURNETT AK, RYDER D, et al. Autologous Peripheral Blood Stem Cell Transplantation in First Remission Adult Acute Myeloid Leukaemia: An Intention to Treat Analysis and Comparison of Outcome Using a Predictive Model Based on the MRC AML10 Cohort. Hematology, 2003; 8 (2), 83-90. http://dx.doi. org/10.1080/1024533031000090793

[7] ZITTOUN RA, MANDELLI F, WILLEMZE R, DE WITTE T, LABAR B, et al. Autologous or allogeneic bone marrow transplantation compared with intensive chemotherapy in acute myelogenous leukemia. N Engl J Med 1995; 332: 217-223. http://dx.doi.org/10.1056/NEJM199501263320403
[8] BURNETT AK, GOLDSTONE AH, STEVENS RMF, HANN IM, REES J KH, et al. Randomized comparison of addition of autologous bone-marrow transplantation to intensive chemotherapy for acute myeloid leukaemia in first remission: results of MRC AML 10 trial. Lancet 1998; 351: 700-708. http://dx.doi.org/10.1016/S0140-6736(97)09214-3

[9] CASSILETH PA, HARRINGTON DP, APPELBAUM FR, LAZARUS HM, ROWE JM, et al. Chemotherapy compared with autologous or allogeneic bone marrow transplantation in the management of acute myeloid leukemia in first remission. $\mathrm{N}$ Engl J Med 1998; 339: 1649-1666. http://dx.doi.org/10.1056/ NEJM199812033392301

[10] APPERLEY J, CARRERAS E, GLUCKMAN E, GRATWOHL A, MASSZI T, editors. Haematopoietic Stem Cell Transplantation, The EBMT Handbook, fifth edition, 2008.

[11] GONDO H, HARADA M, MIYAMOTO T, TAKENAKA $\mathrm{K}$, TANIMOTO K, et al. Autologous peripheral blood stem cell transplantation for acute myelogenous leukemia. Bone Marrow Transplant 1997; 20: 821-826. http://dx.doi. org/10.1038/sj.bmt.1700979

[12] REIFFERS J, LABOPIN M, SANZ M, KORBLING M, BLAISEET D, al. Autologous blood cell vs. marrow transplantation for acute myeloid leukemia in complete remission: an EBMT retrospective analysis. Bone Marrow Transplant 2000; 25: 1115-1119. http://dx.doi.org/10.1038/sj.bmt.1702389

[13] WITTE T DE, KEATING S, SUCIU S, ZITTOUN R, MANDELLI F, et al. A randomized comparison of the value of autologous BMT versus autologous PSCT for patients with AML in first CR in the AML 10 trial of the EORTC LCG and GIMEMA. Blood 2001; 98 (Suppl. 1): 859a (Abstr. 3565).

[14] LINKER CA, RIES CA, DAMON LE, NAVARRO W, RUBIN A, et al. Autologous stem cell transplantation for acute myeloid leukemia in first remission. Biol Blood Marrow Transplant 2000; 6: 50-57. http://dx.doi.org/10.1016/S10838791(00)70052-8

[15] STEIN AS, O`DONNELL MR, SLOVAK ML, SNYDER DS, NADEMANEE AP, et al. Does high dose cytarabine/idarubicin induction followed by autologous stem cell transplant improve survival in adult acute myelogenous leukemia patients with good risk cytogenetics? Blood 2001; 98 (Suppl. 1): 690a (Abstr. 2886).

[16] SLOVAKML, KOPECKY KJ, CASSILETH PA, HARRINGTON $\mathrm{DH}, \mathrm{THEIL} \mathrm{KS}$, et al. Karyotypic analysis predicts outcome of preremission and postremission therapy in adult acute myeloid leukemia: a Southwest Oncology Group/Eastern Cooperative Oncology Group study. Blood 2000; 96: 4075-4083.

[17] SIROHI B, POWLES R, SINGHAL S, MAINWARING P, KULKARNIS, et al. The impact of consolidation chemotherapy on the outcome of autotransplantation for acute myeloid leukemia in first remission; single center experience of 118 adult patients. Blood 2001; 98 (Suppl.1): 690a (Abstr. 2885).

[18] TALLMAN MS, PEREZ WS, LAZARUS HM, GALE RP, MAZIARZ RT, et al. Pretransplant consolidation chemotherapy decreases leukemia relapse after autologous stem cell transplantation for acute myeloid leukemia in first remission. Biol Blood Marrow Transplant. 12: 204-216, 2006. http://dx.doi. org/10.1016/j.bbmt.2005.10.013 